Revista de Neuro-Psiquiatría 2003; 66:296-301

\title{
IMPRESIÓN BASILAR ASOCIADA A MALFORMACIÓN DE ARNOLD-CHIARI EN EL ADULTO
}

\author{
Por CÉSAR A. CASTAÑEDA DÍAZI, ROBERTO PORTILLO VALLENAS ${ }^{\prime}$, \\ JOSÉ VERA RAGGIO' y LUIS DEZA BRINGAS
}

\begin{abstract}
RESUMEN
La malformación de Arnold-Chiari (MACh) es una condición en la cual el cerebelo protruye hacia el canal espinal, puede estar asociada a otras anomalías tales como mielomeningocele, siringomielia y espina bífida. Los síntomas generalmente se inician durante la infancia, o más tardíamente, y se caracterizan por cefalea y signos de disfunción cerebelosa y de otras estructuras de fosa posterior. Presentamos una paciente adulta con esta patología, en la que las manifestaciones más destacadas son cefalea, signos cerebelosos y disfunción del bulbo raquídeo, asociados a fenómeno de impresión basilar. Revisamos las manifestaciones clínicas, diagnóstico, evolución y manejo de esta entidad.
\end{abstract}

\section{ABSTRACT}

In Arnold-Chiari malformation, cerebellum gets into the spinal channel, this condition can be associatted with others anomalies as myelomeningocele, syringomyelia and spina bifida. The clinic manifestations are present during childhood, less frecuently in adolescence and adultness, they usually consist in headache and signs of cerebelar and other posterior fossa structures disfuntion. We report an adult patient with Arnold-Chiari malformation, who presents headache, cerebellar and bulbar disfuntion signs, associated with basilar impression. We review the clinical manifestations, diagnostic, evolution and treatment of this entity.

Palabras-clave: Arnold-Chiari, Neuroimágenes, impresión basilar.

Key WORDS : Arnold-Chiari, Neuroimaging, basilar impression.

1. Neurólogo del Hospital Nacional Guillermo Almenara Irigoyen.

2. Médico residente de Neurología del Hospital Nacional Guillermo Almenara Irigoyen. Persona responsable del artículo:

César A Castañeda Díaz, Jirón Huaraz 1425, Lima-5, PERÚ

Correo electrónico: epsilon54us@yahoo.com 


\section{INTRODUCCIÓN}

La MACh es un trastorno de las estructuras de la fosa posterior en el cual el cerebelo protruye hacia el canal espinal, pudiendo estar asociado con otras anomalías como mielomeningocele, siringomielia y espina bífida. En la mayoría de casos los síntomas se inician durante la infancia; $\sin$ embargo, pueden presentarse en adolescentes y adultos. Los síntomas usualmente incluyen cefalea, vómito, trastorno motor en cara y extremidades, dificultad para deglutir $\mathrm{y}$ varios grados de deterioro mental. Al crecer los niños que tienen la malformación de Chiari, que eran previamente asintomáticos, pueden mostrar signos de disfunción encefálica progresiva, tales como movimientos rápidos involuntarios de los ojos hacia abajo y ataxia. Otros síntomas pueden incluir vértigos, visión doble, sordera, episodios de dolor agudo alrededor de los ojos.

Presentamos un caso a partir del cual se hace la revisión clínica, diagnóstico y tratamiento de tal malformación.

\section{PRESENTACIÓN DEL CASO}

A.A.V., dama de 38 años, inicia sus síntomas a los 29 años en forma insidiosa, con cefalea global y desequilibrio progresivo asociado a lateropulsiones bilaterales, apoyándose para no caer. Posteriormente nota cambios en la voz presentando disfonía caracterizada por sonidos agudos. Pocas semanas antes de su ingreso tiene parestesias y disminución de la fuerza en la parte distal en los miembros inferiores y el dolor de cabeza se torna más intenso y persistente. En el examen clínico se encontró despierta y sin alteraciones cognitivas. Sus funciones vitales y el examen general por sistemas fue normal. Tenía nistagmo provocado horizontal bilateral, reflejo nauseoso disminuido, voz bitonal, abundantes fasciculaciones e hipotrofia de la lengua y cuadriparesia a predominio de miembros inferiores asociada a hipertonía espástica. No se observó atrofia muscular en extremidades ni movimientos involuntarios. Hiperreflexia tendinosa generalizada. Hofmann, Babinski y clonus agotable en forma bilateral. Sensibilidad conservada. Incoordinación motora segmentaria, con severa dismetría, disdiadococinesia y discronometría bilateral.

Los exámenes de laboratorio: hematológicos, lipidograma, ELISA para HTLV-I y VDRL fueron normales. LCR normal. Electromiografría normal. La radiografía cervical mostró fusión de $\mathrm{C} 1$ con occipital. TAC cráneo-cervical convencional y con reconstrucción digital tridimensional reveló agradamiento del espacio de la fosa posterior y del agujero magno e impactación de las apófisis odontoides (impresión basilar) (Figura 1). La RNM de cerebro mostró una malformación de fosa posterior consistente en descenso de amígdalas cerebelosas, atrofia del cerebelo y compresión del tronco cerebral asociado a severo adelgazamiento de la región bulbo-medular (Figura 2).

La paciente fue intervenida quirúrgicamente el 13-12-01, realizándose craneotomía suboccipital, laminectomía de C2 y plastía de meninge. Salió de alta al cabo de dos semanas con mejoría del déficit motor, cervicalgia y paresia del IX par en resolución.

\section{DISCUSIÓN}

La MACh es poco frecuente, De Noronha y colaboradores observaron que la tasa de mayor mortalidad en el período neonatal se debía a malformaciones del sistema nervioso central, de las cuales el $61 \%$ fueron defectos del tubo neural, 8\% evaginación prosencefálica, $3 \%$ de malformaciones de fosa posterior, y sólo 3 casos de MACh. ${ }^{1}$

La MACh comprende un grupo de defectos del SNC en la fosa posterior, las ma- 
nifestaciones clínicas consisten en cefalea, trastornos sensoriales, vértigo, ataxia, alteraciones de la agudeza auditiva, trastornos de nervios craneales bajos, nistagmus, siringomielia, escoliosis, paresia de miembros superiores, hidrocefalia, ápnea, atrofia óptica, heterotopia cerebelar intraorbital, neuralgia o disestesia trigeminal, neuralgia del glosofaríngeo, estados sincopales, disfagia, esotropia, cefalea por hipotensión de LCR, espasmo hemifacial, disfagia y oftalmoplejia internuclear, entre otros. ${ }^{2,3}$ En todos los casos la resonancia magnética por imágenes es fundamental en el diagnóstico.

La malformación del cerebelo es el trastorno común a los 4 tipos descritos por Hans Chiari hace más de 100 años, los cuales, sin embargo, hasta la actualidad carecen de correlato anátomo patológico y embriológico. El tipo I consiste en herniación de las amígdalas cerebelosas en el foramen magno, comprimiendo la unión cráneocervical. Chiari II está casi exclusivamente asociada a mielomeningocele e hidrocefalia, consiste en herniación no sólo de amígdalas cerebelosas sino de todo el contenido de la fosa posterior en el foramen magno. Chiari III y IV son aún menos frecuentes. Chiari III representa un encefalocele. Chiari IV consiste en hipoplasia cerebelosa. Los estudios de Milhorat realizados en 364 pacientes demostraron que la incidencia familiar podría estar a favor de un componente genético de transmisión. ${ }^{4}$

La MACh de tipo I tiene el inicio más tardío de los síntomas, y es la variante de nuestro trabajo. La paciente refirió haber tenido cefalea desde la niñez, cuyo mecanismo pudo ser la distensión de la fosa posterior. Stovner describió casos con cefalea de presentación episódica y otros de forma prolongada y continua en ocasiones ésta era desencadenada por la tos y otras maniobras de Valsalva, diferenciándose así de la migraña y la cefalea cervicogénica. ${ }^{5}$ Nuestra paciente presentó incoordinación cerebelosa progresiva asociada a signos piramidales, que se pueden atribuir a lesión atrófica del cerebelo y desplazamiento del tronco cerebral hacia el canal raquídeo, con trastornos de IX y XII pares craneales, constituyendo el síndrome bulbar anterior, teniendo importancia el efecto compresivo de la apófisis adontoides impactada.

La patogenia no está bien esclarecida, ${ }^{6}$ aunque se ha planteado que se debe a una desproporción entre las estructuras posteriores del encéfalo y las dimensiones de la fosa posterior, al respecto se han realizado mediciones con ayuda de la RMN para determinar las proporciones entre la estructura craneal y el encéfalo posterior, sin embargo, se ha llegado a la conclusión que los factores responsables para la aparición de los síntomas siguen siendo poco conocidos. Aunque los estudios de Christophe y colaboradores, realizado en base a mediciones del volumen de fosa posterior en pacientes y controles sanos, llegan a la conclusión que la MACh se debe a un defecto de desarrollo del hueso occipital originado del mesodermo paraaxial, lo que provoca estrechamiento de la fosa posterior del cráneo, que contiene el cerebro posterior desarrollado normalmente. ${ }^{7}$

En estos casos está indicada la cirugía, a pesar de la posibilidad de disfunción neurológica consistente en siringomielia sintomática o hidrocéfalo, observado en el $15 \%$ de los casos. El pronóstico de estos casos es variable, dependiendo de los síntomas preexistentes y la respuesta a la cirugía con craneotomía suboccipital, laminectomía de $\mathrm{C} 2$ e injerto dural, además de reducción del volumen de las amígdalas cerebelosas. $^{8}$ 


\section{FiguRA 1}
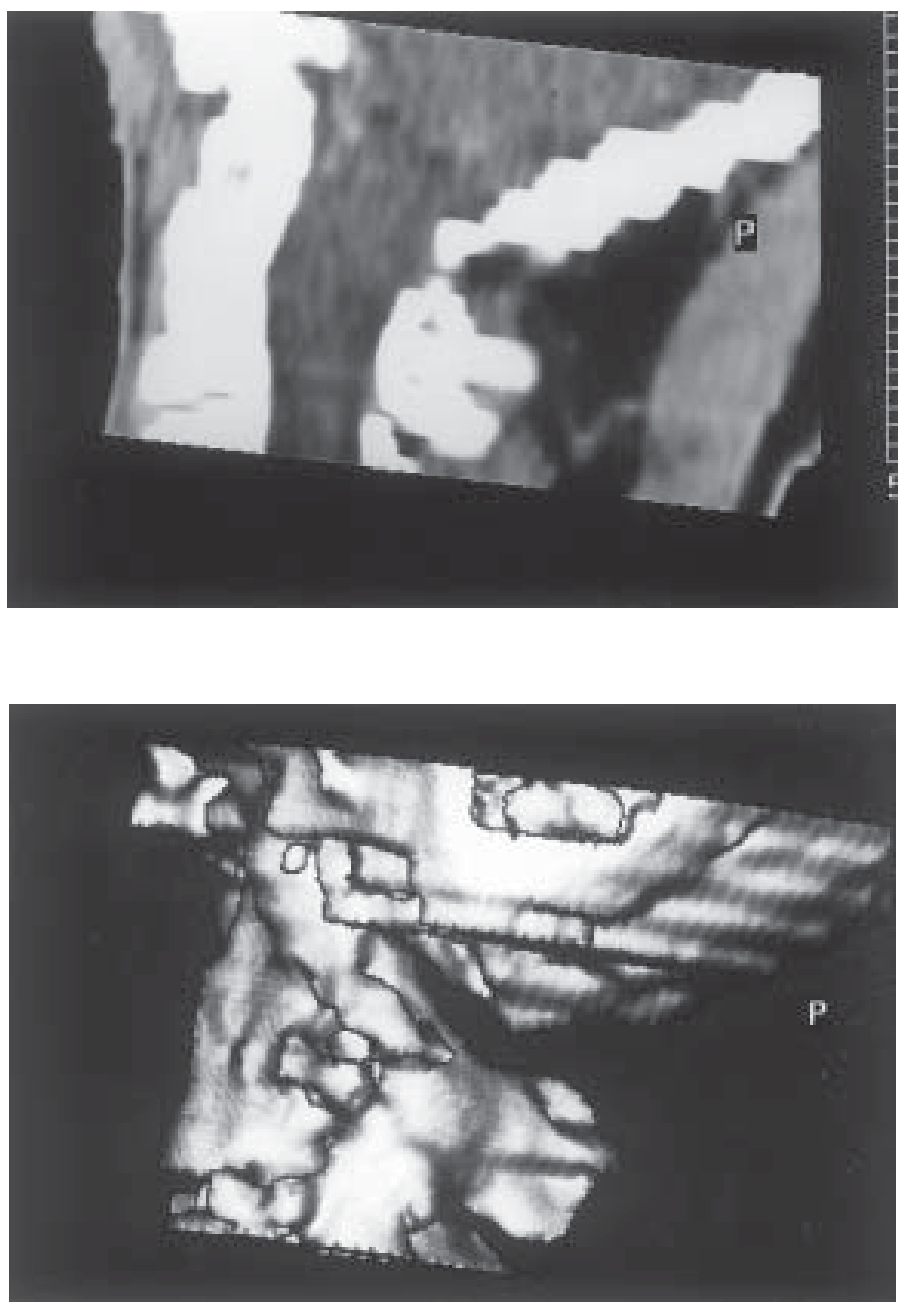

Tomografía axial computarizada cráneo-cervical convencional y con reconstrucción tridimensional: agrandamiento del espacio de la fosa posterior y el agujero magno, e impresión basilar. 


\section{FiguRA 2}
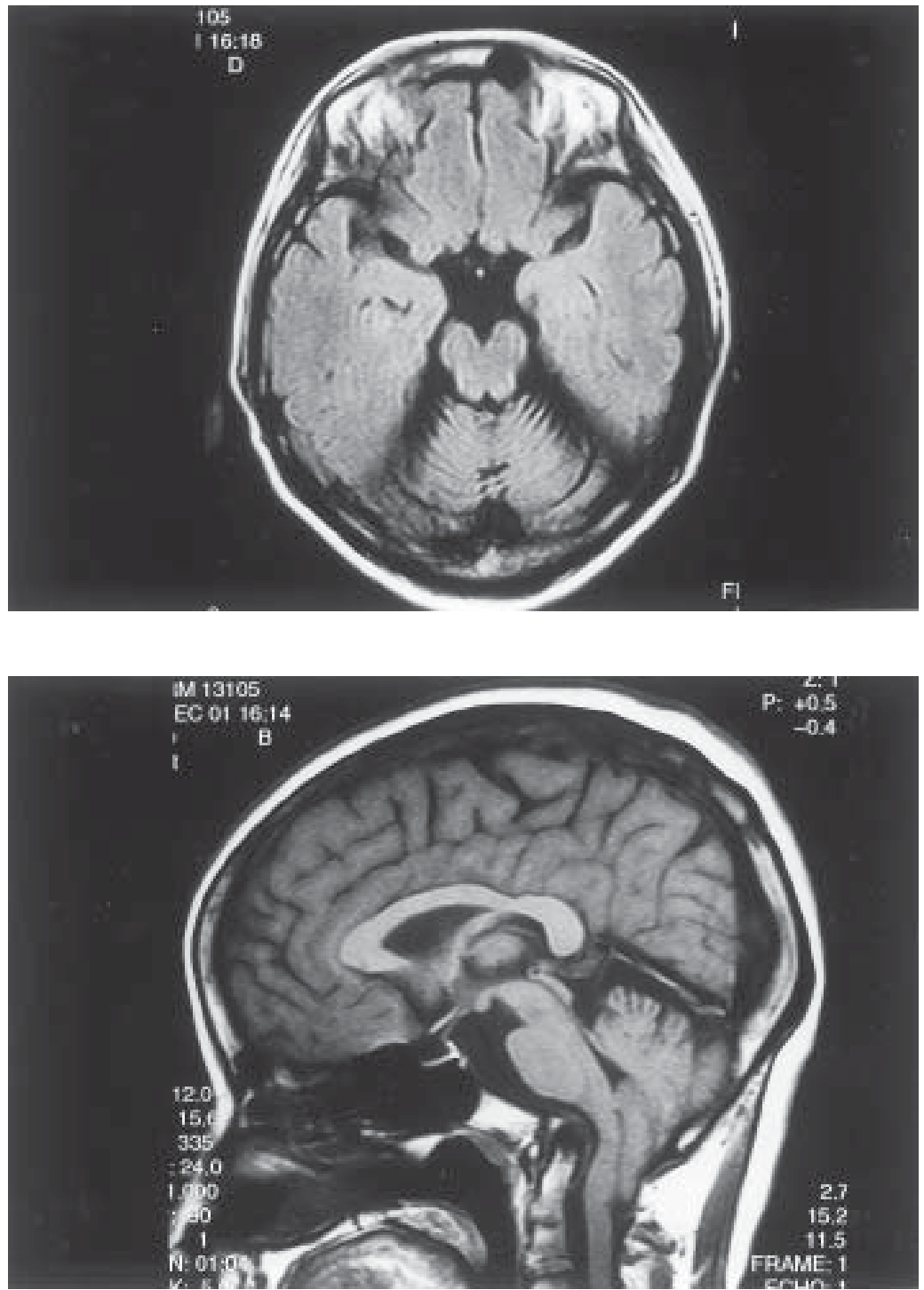

Resonancia magnética por imágenes de cerebro: malformación de fosa posterior consistente en descenso de amígdalas cerebelosas, atrofia del cerebelo y compresión del tronco cerebral con severo adelgazamiento del bulbo. 


\section{CONSIDERACIONES FINALES}

La MACh es una condición en la cual el cerebelo protruye hacia el canal espinal, pudiendo estar asociada con otras anomalías como mielomeningocele, siringomielia y espina bífida.

En este trastorno la mayoría de los síntomas frecuentemente se inician durante la infancia, sin embargo, pueden presentarse en la adolescencia o tardíamente en el adulto.

Los síntomas usualmente incluyen cefalea, dificultad para deglutir y varios grados de déficit motor de extremidades. Pueden coexistir signos de disfunción cere- bral progresiva, tales como movimientos rápidos involuntarios de los ojos hacia abajo, vértigos, visión doble, sordera y deterioro en la habilidad para coordinar los movimientos.

Presentamos una paciente, mujer de 38 años, que padeció de cefalea crónica, incoordinación cerebelosa progresiva y signos de disfunción del bulbo raquídeo como consecuencia de tener impresión basilar y otros defectos congénitos del sistema nervioso, conocidos con el nombre de malformaciones de Arnold Chiari I.

El tratamiento quirúrgico consistente en craneotomía suboccipital, laminectomía de C2 y plastía de meninge.

\section{BIBLIOGRAFÍA}

1. De Noronha L, Medeiros F, Martins VD, Sampaio GA, Serapiao JM, Kastin $G$, Torres. Malformations of the central nervous system: analysis of 157 pediatric autopsies. Arq Neuropsiquiatr $2000 \mathrm{Sep}$; 58(3B): 890-6.

2. Yamazaki Y, Tachibana S, Takano M, Fujii K. Clinical and neuroimaging features of Chiari type I malformations with and without associated syringomyelia. Neurol Med Chir (Tokyo). 1998 Sep; 38(9): 541-6.

3. Storrs TJ, Roberts CI. Adult Chiari malformation with headache and trigeminal dysest. Oral Surg Oral Med Oral Pathol Oral Radiol Endod. 1996 Sep; 82(3):284-7.

4. Milhorat TH, Chou MW, Trinidad EM, Kula RW, Mandell M, Wolpert C, Speer MC. Chiari I malformation redefined: clinical and radiographic findings for 364 symptomatic patients. Neurosurgery. 1999 May; 44(5): 1005-17.

5. Stovner LJ. Headache associated with the Chiari type I malformation. Headache. 1993 Apr;33(4): 175-81.

6. Christophe C, Dan B. Magnetic resonance imaging cranial and cerebral dimensions: is there a relationship with Chiari I malformation? A preliminary report in children. Europ J Paediatr Neurol. 1999; 3(1): 15-23.

7. Niskikawa M, Sakamoto H, Hakuba A, Nakanishi N, Inoue Y. Pathogenesis of Chiari malformation: a morphometric study of the posterior cranial fossa. J Neurosurg. 1997 Jan; 86(1):40-7.

8. Strayer A. Chiari I malformation: clinical presentation and management. J Neurosci Nurs. 2001 Apr; 33(2): 90-6, 104. 